\title{
The combined effect of aortic stiffness and pressure wave reflections on mortality in the very old with cardiovascular disease: the PROTEGER Study
}

\author{
Athanase D Protogerou ${ }^{1}$, Michel E Safar ${ }^{2}$, Theodoros G Papaioannou ${ }^{3}$, Yi Zhang ${ }^{2}$, Davide Agnoletti ${ }^{2}$, \\ Dimitrios Papadogiannis ${ }^{1}$ and Jacques Blacher ${ }^{2}$
}

Aortic stiffness (pulse wave velocity, PWV) and pressure wave reflections (augmentation index, Al) are two interrelated markers of mortality. Their potential synergistic effect on mortality has never been studied. We sought to investigate the association as well as the combined effect of PWV and AI on mortality in a cohort (PROTEGER Study) of very old ( $>70$ years, mean age ( \pm s.d.): $85.4 \pm 7.4$ years). Aortic PWV and pressure wave AI were assessed by pulse wave analysis; carotid structure and cardiac function were analyzed by ultrasound. The analysis was performed in 259 patients who had all the data available. Neither PWV nor Al was, in separate, predictors of mortality (log-rank test: $P=0.278$ and $P=0.433$, respectively, Kaplan-Meier analysis). No linear association was found between PWV and AI $(P=0.278)$. To investigate the possible synergistic effect of PWV and Al on mortality, the population was analyzed according to the tertiles of PWV and Al. Subjects with combined high PWV (third tertile) and moderate-to-high Al (second and third tertiles) had lower survival compared with the rest of the population (Kaplan-Meier survival curve, log-rank test: $\boldsymbol{P}=\mathbf{0 . 0 3 0}$ ). Cox regression analysis showed that this effect was independent from age, gender, blood pressure, cardiac/carotid parameters and other confounders, except creatinine and insulin resistance. The present study provides further insight on the pathophysiological association between large artery stiffness and pressure wave reflections, suggesting that when both are elevated they may increase the mortality in the elderly.

Hypertension Research (2011) 34, 803-808; doi:10.1038/hr.2011.33; published online 7 April 2011

Keywords: arterial stiffness; elderly; mortality; pressure wave reflections

\section{INTRODUCTION}

In elderly populations, traditional cardiovascular $(\mathrm{CV})$ risk factors are known to be less informative regarding the prediction of mortality. ${ }^{1,2}$ The PROTEGER Study was designed to detect markers (arterial, cardiac, metabolic or nutritional) that further provide prognostic information on the survival of elderly subjects with $\mathrm{CV}$ disease or high CV risk. Aortic stiffness, assessed by the carotid-femoral pulse wave velocity (PWV), is a robust predictor of mortality in the general as well as in the elderly population. ${ }^{3-5}$ In the PROTEGER Study, no association between carotid-femoral PWV and mortality was found. ${ }^{6,7}$ Augmentation index (AI), a marker of pressure wave reflections, is also an independent predictor of mortality. ${ }^{8}$ The current theory of wave reflections suggests that $\mathrm{AI}$ is positively associated with aortic PWV. This association may represent a mechanism, which is at least partly responsible for the deleterious effects of aortic stiffening, as it leads to increased left ventricular afterload. However, it is not consistent; it is disrupted by aging, ${ }^{9,10}$ inflammation ${ }^{11}$ or insulin resistance. $^{12}$
In the present study, our aim was to investigate the potential synergistic effect of aortic PWV and AI on mortality, which has never been investigated before. We tested whether the classical linear association between these two hemodynamic parameters (PWV and AI) is disrupted in the frail and elderly population of the PROTEGER Study, as previously described in other cohorts. ${ }^{9}$ Moreover, we performed a post hoc analysis to investigate the potential synergistic effect of PWV and AI (as assessed by dividing the population in tertiles of PWV and AI) on mortality, independently from blood pressure (BP) level and other confounders.

\section{METHODS}

Study cohort

From May 2000 to November 2001, all patients entering the Geriatric Departments of Charles Foix and Emile Roux Hospitals, Ile de France, and meeting the following inclusion criteria: age $>70$ years old, history of $\mathrm{CV}$ disease (including coronary heart disease, cerebro-vascular disease or any other $\mathrm{CV}$ events of the upper or lower limbs, thoracic or abdominal aorta, or renal

\footnotetext{
${ }^{1}$ Hypertension Center and Cardiovascular Research Laboratory, 1st Department of Propaedeutic and Internal Medicine, Laiko Hospital, Medical School, National and Kapodistrian University of Athens, Athens, Greece; ${ }^{2}$ Diagnosis and Therapeutic Center, AP-P, Hopital Hotel-Dieu, Paris-Descartes University Faculty of Medicine, Paris, France and ${ }^{3}$ Biomedical Engineering Unit, 1st Department of Cardiology, Hippokration Hospital, Medical School, National \& Kapodistrian University of Athens, Greece

Correspondence: Professor ME Safar, Paris-Descartes University, Faculty of Medicine, Hôpital Hôtel-Dieu, Diagnosis and Therapeutic Center, 1, place du Parvis Notre-Dame, 75181 Paris Cedex 04, France.

E-mail: michel.safar@htd.aphp.fr
}

Received 11 August 2010; revised 27 January 2011; accepted 30 January 2011; published online 7 April 2011 
arteries) or hypertension, mini mental status examination $>15 / 30$, absence of fatal disease with life expectancy $<1$ month, willingness to give a written informed consent to participate in this study were included in the PROTEGER Study. Patients with cachexia (body mass index, BMI $<17 \mathrm{~kg} \mathrm{~m}^{-2}$ ) and/or evolutive cancer and/or advanced renal failure (plasma creatinine $>250 \mu \mathrm{moll}^{-1}$ ) were excluded from the study.

The study cohort was then composed of 331 subjects (86 men and 245 women) with mean age ( \pm s.d.) of $85 \pm 7$ years. Approximately $13 \%$ were hospitalized because of dementia or neuropsychological problems, 35\% because of traumatism, $23 \%$ because of CV disease event, $9 \%$ because of infectious disease, $9 \%$ because of social reasons and $11 \%$ for other miscellaneous causes. The PROTEGER Study was approved by the Committee for the Protection of Human Subjects in Biomedical Research of Saint Germain Hospital (Ile de France). Written informed consent was obtained from all participants, after informing them as well as their relatives.

Only the parameters that were relevant to the present analysis are presented below.

\section{Social, anthropometric and clinical parameters}

Information compiled from the questionnaire filled out at inclusion included gender, age, weight and height, history of $\mathrm{CV}$ event, the presence of diabetes mellitus, dyslipidemia, hypertension, smoking habits and previous diseases. The reason for hospitalization as well as the level of education (1: primary school, 2: college degree, 3: bachelor degree, 4: university degree) was registered.

\section{Medications}

Antihypertensive drugs included: diuretics (38.0\%), calcium channel antagonists $(27.9 \%)$, angiotensin-converting enzyme inhibitors $(26.1 \%), \beta$-blockers (12.3\%), $\alpha$-blockers $(4.0 \%)$ and central acting agents $(3.1 \%)$, either alone or in combination. Approximately 3\% of the patients were medically treated for dyslipidemia (drugs, including statins or fibrates) and 14\% for diabetes mellitus (drugs, including sulfonamides and/or biguanids, or insulin).

\section{Assessment of BP, arterial stiffness and pressure wave reflections}

The measurements were performed in the morning, after an overnight fast, in supine position. Brachial BP was measured after $15 \mathrm{~min}$ rest, using the semiautomatic oscillometric device, Dynamap (Kontron, Paris, France). Five measurements, 2 min apart, were averaged.

AI was assessed by means of applanation tonometry and by application of pulse wave analysis (PWA) at the level of the carotid artery; the carotid pressure waveform was calculated as previously described. ${ }^{6,13}$

Aortic PWV was determined using the foot-to-foot method as previously described $^{6}$ (Complior, Colson, Paris, France). The superficial distance covered by the pulse wave was measured directly from the carotid to the femoral artery.

\section{Measurement of carotid and cardiac ultrasound parameters}

The presence of carotid plaques was assessed by a high-resolution B-mode (7.5 MHz transducer, Kontron 440). Echocardiograms were recorded with an ultrasound system (Kontron 440 ) using a $2.5-\mathrm{MHz}$ phased-array transducer. Cardiac measurements were performed according to the American Society of Echocardiography by M-mode measurements (LV mass: 0.8 (1.04 ([LVIDD+ PWTD+IVSTD $\left.\left.\left.]^{3}-[\text { LVIDD }]^{3}\right)\right)+0.6 \mathrm{~g}\right)$ where $\mathrm{LVIDD}=$ left ventricular internal diameter in diastole; PWTD=posterior wall thickness in diastole; IVSTD= interventricular septum thickness in diastole. It was possible to evaluate left ventricular volumes only with left ventricular diameters $(D)$ assuming that the geometric shape of the ventricle was an ellipse. Then the volume of this ellipse was expressed as: $V=(4 \pi / 3)(2 D / 2)(D / 2)(D / 2)=\pi D^{3} / 3 \approx D^{3}$. Cardiac output $(Q)$ was calculated with the formula: stroke volume $(\mathrm{SV}) \times$ heart rate. Total peripheral vascular resistance (TPR), as: $\mathrm{TPR}=\mathrm{MBP} / \mathrm{Q}$. Echocardiograms were also used to evaluate the diastolic index: E wave deceleration slope time.

\section{Measurement of biological parameters}

Venous blood samples were obtained in subjects after an overnight fast. Plasma was separated without delay at $4^{\circ} \mathrm{C}$ in a refrigerated centrifuge and stored at $4^{\circ} \mathrm{C}$ (for the determination of routine chemistry profile by standard methods) until analysis. Total cholesterol and triglycerides were determined with the use of a Technicon Chem assay (Technicon Instruments), and HDL cholesterol was measured in the supernatant after precipitation of apolipoprotein B-containing lipoproteins with heparin-manganese chloride. Low-density lipoprotein cholesterol was calculated by the formula of Friedewald for patients with serum triglyceride concentrations $<4.0 \mathrm{mmoll}^{-1}$. Blood cells counts were determined (Argos 3, ABX, Montpellier, France), and the plasma creatinine (Hitachi 911 analyzer with Roche reagents, Roche Hitachi, Paris, France) levels were measured. Plasma albumin was determined using immunonephelometric methods on Immage system (Beckman Coulter, Villepinte, France).

\section{Follow-up procedures}

Follow-up started from the baseline examination and lasted until April 2004. Information was obtained from the patient himself, from relatives or from general practitioners. Interim telephone and clinic contacts were used to assess all hospitalizations and outpatient CV diagnoses, and overall mortality. In case of hospitalization, discharge reports from medical specialists were obtained. Follow-up time was defined as the time from the baseline visit until the first event (for those who had an event) or it was censored at the last contact date (for those who did not have any event or for the three patients that were lost to follow-up). Of all 331 participants in the present study, 3 were lost to followup; the mean duration of follow up was $380 \pm 196$ days.

\section{Statistics}

The current analysis was performed only in those subjects who had all the needed data available $(n=259)$. Linear regression analysis was used to detect the presence of association between AI and PWV. To investigate the incidence of mortality according to the level of PWV and AI, we divided the population according to tertiles of PWV and AI. Kaplan-Meier survival curve analysis and log-rank test were applied to detect differences in the survival of the patients according to the level of AI or PWV independently or in combination. Cox regression analysis was applied to investigate the combined effect AI and PWV on mortality independently from other confounding factors. Statistical tests such as $t$ - and $\chi^{2}$-tests, as appropriate, were applied to detect the presence of differences regarding CV risk factors, drugs, metabolic, biochemical and inflammatory parameters between subgroups according to the level of AI and PWV. Analysis of covariance was applied to detect differences in hemodynamic, cardiac and vascular parameters among subgroups of the population, after adjustment for covariates. Statistical analysis was performed on a SPSS 11.5 version. A $P<0.05$ was considered statistically significant.

\section{RESULTS}

Out of the 259 subjects, 87 (33.6\%) died during the follow-up period. Their demographics are provided in Table 1.

In total population, no linear association was found between $\mathrm{AI}$ and PWV ( $r=-0.068, P=0.278$ ). Neither AI (divided in tertiles) nor PWV (divided in tertiles) was associated with mortality (log-rank test for total mortality by Kaplan-Meier analysis: $P=0.433$ and $P=0.192$, respectively).

Mortality according to the level (tertile) of PWV and AI is provided in Table 2. Subjects in the third tertile of PWV (higher level of stiffness) had higher than the average $(>33.6 \%)$ mortality, except from those who had at the same time low AI (first tertile of AI). On the contrary, subjects in the third tertile of AI (higher level of wave reflections) had lower than average $(<33.6 \%)$ mortality, except from those who had at the same time very high PWV (third tertile of PWV).

We further explored the presence of potential excess mortality in subjects with both impaired PWV and AI as suggested by Table 2 (gray boxes: group A). Kaplan-Meier survival curve analysis showed that group A had higher overall mortality than the rest of the population (group B), log-rank $P=0.030$ (Figure 1). When performing KaplanMeier analysis for non-CV $(n=60)$ and for established CV $(n=27)$ death separately, we found a similar (but nonsignificant) trend $(P=0.115$ and $P=0.123$, respectively). 
Table 1 Cardiovascular risk factors, drug treatment and biochemical parameters in total population

\begin{tabular}{|c|c|}
\hline & Total population \\
\hline Cardiovascular risk factors & $N=259$ \\
\hline Age (years) & $85.4 \pm 0.4$ \\
\hline Gender (\%, females) & 74.1 \\
\hline Educational level: primary school (\%) & 62.9 \\
\hline Educational level: college degree (\%) & 23.6 \\
\hline Educational level: bachelor degree (\%) & 6.2 \\
\hline Educational level: university degree (\%) & 7.3 \\
\hline Mini mental scale & $73.9 \pm 17.2$ \\
\hline Weight (kg) & $60.4 \pm 13.5$ \\
\hline Diabetes mellitus (\%) & 19.7 \\
\hline Dyslipidemia (\%) & 17.4 \\
\hline Hypertension (\%) & 75.7 \\
\hline Normal sinus rhythm (\%) & 79.4 \\
\hline Current smokers (\%) & 5.0 \\
\hline Ex-smokers (\%) & 20.9 \\
\hline History of stroke (\%) & 29.5 \\
\hline History of coronary heart disease (\%) & 31.8 \\
\hline \multicolumn{2}{|l|}{ Drug treatment } \\
\hline Total anti-HTN drugs (s.d.) & $1.2 \pm 0.1$ \\
\hline Diuretics (\%) & 37.1 \\
\hline A-blockers (\%) & 4.2 \\
\hline B-blockers (\%) & 11.2 \\
\hline Central acting agents (\%) & 2.3 \\
\hline Calcium channel antagonists (\%) & 28.2 \\
\hline ACE inhibitors (\%) & 25.5 \\
\hline \multicolumn{2}{|l|}{ Biochemical parameters } \\
\hline Hematocrit (\%) & $36.2 \pm 0.4$ \\
\hline Albumin $\left(\mathrm{g} \mathrm{dl}^{-1}\right)$ & $3.4 \pm 0.5$ \\
\hline Glucose $\left(\mathrm{mg} \mathrm{dl}^{-1}\right)$ & $105.8 \pm 12.2$ \\
\hline Total cholesterol $\left(\mathrm{mg} \mathrm{dl}^{-1}\right)$ & $206.2 \pm 2.9$ \\
\hline LDL cholesterol (mg dl-1) & $134.0 \pm 2.6$ \\
\hline HDL cholesterol ( $\left.\mathrm{mg} \mathrm{dl}^{-1}\right)$ & $43.3 \pm 0.8$ \\
\hline Triglycerides $\left(\mathrm{mg} \mathrm{dl}^{-1}\right)$ & $141.1 \pm 5.3$ \\
\hline
\end{tabular}

Abbreviations: ACE, angiotensin-converting enzyme; CRP, C-reactive protein; HDL, high-density lipoprotein; HTN, hypertension; LDL, low-density lipoprotein.

Education level, 1 to 4 (see text 'Methods section' for details).

To investigate the potential causes that may lead to higher overall mortality in subjects with high PWV combined with moderateto-high AI (group A), we compared by independent groups $t$-test and $\chi^{2}$-test (Table 3 ) the potential confounding factors between group A and group B. No significant differences regarding: age, gender, weight, educational status, mini mental scale, as well as regarding the presence of diabetes mellitus, hypertension, arrhythmia, smoking, renal function, history for stroke and coronary heart disease, the use of drugs (except from calcium channel antagonists, $P=0.037$ ), as well as metabolic and biochemical were observed. Similarly we tested for differences between group A and group B regarding hemodynamic and vascular parameters (Table 4). Group A had significantly higher SBP, DBP, MBP, PP, PWV (even after adjustment for age, gender, MBP), AI (even after adjustment for age, gender, HR), as well as lower HR and TPR. Cardiac and carotid parameters (LV mass, EF, diastolic function, percentage of carotid plaques) were similar between the two groups.

Cox regression analysis (Table 5) showed that subjects with both high PWV and moderate-to-high AI (group A) had (independent of
Table 2 Each box depicts the incidence of mortality (\%, that is deceased subjects/total number) according to the level (tertiles) of augmentation index (AI) and pulse wave velocity (PWV). Boxes in gray color (group $\mathrm{A}$ ) have percentage of mortality higher than the average value (that is, $>33.6 \%$ )

\begin{tabular}{|c|c|c|c|}
\hline & \multicolumn{3}{|c|}{ PWV tertiles } \\
\hline & $\begin{array}{c}\mathrm{I}<12.48 \\
\mathrm{~m} \mathrm{sec}^{-1}\end{array}$ & $\begin{array}{c}\text { II } \\
\left(12.49-15.17 \mathrm{~m} \mathrm{sec}^{-1}\right)\end{array}$ & $\begin{array}{c}I I I \\
\left(>15.18 \mathrm{msec}^{-1}\right)\end{array}$ \\
\hline \multicolumn{4}{|l|}{ Al tertiles } \\
\hline I (<111\%) & $37.5(9 / 24)$ & $25.8(8 / 31)$ & $33.3(11 / 33)$ \\
\hline II (112-126\%) & $29.0(9 / 31)$ & $30.4(7 / 23)$ & $53.1(17 / 32)$ \\
\hline III (>127\%) & $24.1(7 / 29)$ & $28.1(9 / 32)$ & $41.6(10 / 24)$ \\
\hline
\end{tabular}

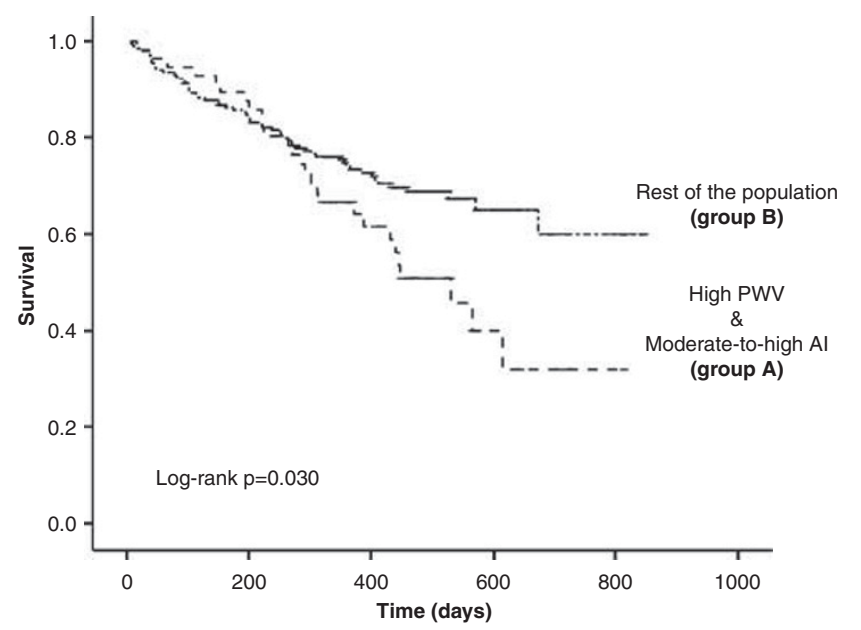

Figure 1 Kaplan-Meier survival curve analysis and log-rank test were applied to detect differences in the survival of the patients according to the level of pulse wave velocity (PWV) and augmentation index (Al), as defined in Table 2.

age, gender, center effect, heart rate and MBP) higher mortality than the rest of the population (group B). Similar results were found when MBP was replaced by DBP or SBP or PP (these data are not shown). The same result was also observed after using (one step at a time) different models for additional adjustment regarding: mental status, history of stroke and coronary heart disease, CV risk factors (DM, smoking, hypercholesterolemia), nutritional status, cardiac parameters (LV mass, EF, LV diastolic function), drug treatment (data not shown) and carotid atherosclerosis as assessed by the presence of carotid plaques (data not shown).

After adjustment for renal function (creatinine), the statistical significance of the effect of high arterial stiffness and moderate-tohigh wave reflections on mortality was marginally lost $(P=0.055$, model 7, Table 5).

When subjects with diabetes mellitus $(n=51)$ or when subjects under insulin treatment $(n=17)$ were excluded from the analysis, the statistical significance of the effect of high arterial stiffness and moderate-to-high wave reflections on mortality were also marginally lost (data not shown).

\section{DISCUSSION}

In the present study, three main findings merit further discussion: (1) the classically observed linear association between PWV and AI was disturbed; (2) the mortality was associated with age, gender, DBP, CV 
Table 3 Cardiovascular risk factors, drug treatment and biochemical parameters in subjects with combined high pulse wave velocity (PWV) (third tertile) and moderate-to-high augmentation index (AI) (second and third tertiles), (group A). Group B represents the rest of the population

\begin{tabular}{|c|c|c|c|}
\hline Cardiovascular risk factors & $\begin{array}{c}\text { Group A } \\
N=56\end{array}$ & $\begin{array}{l}\text { Group B } \\
N=203\end{array}$ & P-value* \\
\hline Age (years) & $85.9 \pm 7.7$ & $85.3 \pm 6.8$ & 0.608 \\
\hline Gender (\%, males) & 73.4 & 76.8 & 0.608 \\
\hline Educational level: primary school (\%) & 62.6 & 64.3 & \\
\hline Educational level: college degree (\%) & 21.7 & 30.4 & \\
\hline Educational level: bachelor degree (\%) & 7.4 & 1.8 & \\
\hline Educational level: university degree (\%) & 8.4 & 3.6 & 0.169 \\
\hline Mini mental scale & $73.9 \pm 18.4$ & $73.8 \pm 16.4$ & 0.947 \\
\hline Weight (kg) & $59.7 \pm 14.3$ & $60.6 \pm 13.3$ & 0.669 \\
\hline Diabetes mellitus (\%) & 21.4 & 19.2 & 0.712 \\
\hline Dyslipidemia (\%) & 8.9 & 19.8 & 0.072 \\
\hline Hypertension (\%) & 75.0 & 75.9 & 0.894 \\
\hline Normal sinus rhythm (\%) & 79.6 & 79.3 & 0.955 \\
\hline Current smokers (\%) & 7.3 & 4.4 & 0.547 \\
\hline Ex-smokers (\%) & 18.2 & 21.7 & 0.623 \\
\hline History of stroke (\%) & 30.9 & 29.1 & 0.790 \\
\hline History of coronary heart disease (\%) & 26.8 & 33.2 & 0.364 \\
\hline \multicolumn{4}{|l|}{ Drug treatment } \\
\hline Total anti-HTN drugs (\%) & $1.4 \pm 1.0$ & $1.2 \pm 1.0$ & 0.210 \\
\hline Diuretics (\%) & 42.9 & 35.5 & 0.311 \\
\hline$\alpha$-Blockers (\%) & 3.6 & 4.4 & 0.999 \\
\hline$\beta$-Blockers (\%) & 8.9 & 11.8 & 0.543 \\
\hline Central acting agents (\%) & 3.6 & 2.0 & 0.613 \\
\hline Calcium channel antagonists (\%) & 39.3 & 25.1 & 0.037 \\
\hline ACE inhibitors (\%) & 30.4 & 24.1 & 0.344 \\
\hline Insulin (\%) & 5.4 & 10.7 & 0.217 \\
\hline \multicolumn{4}{|l|}{ Biochemical parameters } \\
\hline Hematocrit (\%) & $36.6 \pm 4.6$ & $36.1 \pm 7.1$ & 0.621 \\
\hline Albumin $\left(\mathrm{g} \mathrm{dl}^{-1}\right)$ & $3.5 \pm 0.4$ & $3.4 \pm 0.5$ & 0.333 \\
\hline Creatinine $\left(\mathrm{mg} \mathrm{dl}^{-1}\right)$ & $1.01 \pm 0.5$ & $0.91 \pm 0.5$ & 0.071 \\
\hline Glucose $\left(\mathrm{mg} \mathrm{dl}^{-1}\right)$ & $107.3 \pm 11.2$ & $105.5 \pm 12.1$ & 0.810 \\
\hline Total cholesterol $\left(\mathrm{mg} \mathrm{dl}^{-1}\right)$ & $211.3 \pm 49.8$ & $204.8 \pm 46.2$ & 0.375 \\
\hline LDL cholesterol (mg dl-1) & $135.9 \pm 45.1$ & $133.6 \pm 38.1$ & 0.719 \\
\hline HDL cholesterol $\left(\mathrm{mg} \mathrm{dl}^{-1}\right)$ & $45.3 \pm 11.8$ & $42.8 \pm 12.1$ & 0.175 \\
\hline Triglycerides (mg dl-1) & $161.0 \pm 141.9$ & $135.8 \pm 60.2$ & 0.211 \\
\hline
\end{tabular}

Abbreviations: ACE, angiotensin-converting enzyme; CRP, C-reactive protein; HDL, high-density lipoprotein; HTN, hypertension; LDL, low-density lipoprotein.

Education level, 1 to 4 (see text 'Methods section' for details).

* $P$ indicates significance of differences between groups: A vs. B. Dichotomous variables are expressed as percentages ( $\chi^{2}$-test) and continuous variables as mean \pm s.d. ( $t$-test).

disease, denutrition (low albumin) and renal function, but neither with elevated aortic stiffness (carotid to femoral PWV) nor with pressure wave reflections (AI), when these two parameters were evaluated alone; (3) the combination of high PWV and moderateto-high AI, but not the opposite (that is, high AI combined with moderate-to-high PWV), was a predictor of mortality independently age, gender and BP. Renal function and insulin resistance seem to modulate the effect of combined effect of PWV and AI on mortality.

The population of the PROTEGER Study has several particularities. It is composed mainly of 'survivors' and a high prevalence of overt atherosclerotic disease (almost 62\%). Only 80 subjects were younger than 80 years of age and 131 were over 90 years old (mean age 85.4
Table 4 Comparison of the hemodynamic, cardiac and vascular parameters between subjects with combined high PWV (third tertile) and moderate-to-high AI (second and third tertiles, group A) vs the rest of the population (group B)

\begin{tabular}{lccr}
\hline & Group A & Groups B & \\
& $N=56$ & $N=203$ & P-value* \\
& & & \\
\hline Heart rate (b.p.m.) & $68.2 \pm 9.4$ & $71.3 \pm 12.0$ & $\mathbf{0 . 0 4 3}$ \\
Peripheral systolic BP (mm Hg) & $145.4 \pm 20.3$ & $129.3 \pm 19.0$ & $<\mathbf{0 . 0 0 1}$ \\
Peripheral diastolic BP (mm Hg) & $68.6 \pm 12.4$ & $64.5 \pm 11.3$ & $\mathbf{0 . 0 1 8}$ \\
Peripheral PP (mm Hg) & $76.9 \pm 15.7$ & $64.8 \pm 15.2$ & $<\mathbf{0 . 0 0 1}$ \\
Peripheral mean BP (mm Hg) & $100.7 \pm 19.0$ & $89.4 \pm 13.7$ & $<\mathbf{0 . 0 0 1}$ \\
Carotid systolic BP (mm Hg) & $138.6 \pm 18.0$ & $121.3 \pm 19.5$ & $<\mathbf{0 . 0 0 1}$ \\
TPR (arbitrary unit) & $19.2 \pm 6.9$ & $16.9 \pm 6.7$ & $\mathbf{0 . 0 3 3}$ \\
Carotid PP (mm Hg) & $64.4 \pm 16.4$ & $50.3 \pm 15.5$ & $<\mathbf{0 . 0 0 1}$ \\
Carotid Alx (\%)** & $125.6 \pm 13.8$ & 115.524 .9 & $\mathbf{0 . 0 0 3}$ \\
Carotid-femoral PWV (m s ${ }^{-1}$ ) & $18.3 \pm 2.8$ & $13.4 \pm 3.0$ & $<\mathbf{0 . 0 0 1}$ \\
Carotid plaques (\%) & 96.0 & 94.6 & 0.326 \\
Ejection fraction (\%) & $61.2 \pm 12.7$ & $61.2 \pm 11.6$ & 0.999 \\
Stroke volume (ml) & $84.3 \pm 23.4$ & $83.3 \pm 24.5$ & 0.797 \\
LV mass (g) & $186.5 \pm 61.2$ & $185.1 \pm 69.4$ & 0.897 \\
Deceleration time (ms) & $210.9 \pm 84.2$ & $207.3 \pm 63.5$ & 0.730 \\
\hline
\end{tabular}

Abbreviations: Al, augmentation index; AP, augmented pressure; BP, blood pressure; LV, left ventricle; PP, pulse pressure; PWV, pulse wave velocity, TPR, total peripheral resistance. ${ }^{*} P$ indicates significance of differences between groups: A vs. B. Variables are expressed as mean \pm s.d.

**Adjusted for heart rate

years (70-103)). Carotid to femoral PWV was consistently augmented, over passing the $20 \mathrm{~m} \mathrm{sec}^{-1}$ in almost $10 \%$ of the population. However, only $10 \%$ of the subjects had seriously impaired left ventricular function (ejection fraction $<45 \%$ ). These major traits of the population may be responsible for a number of CV particularities and results, which must be carefully considered and may limit the extrapolation of our results to other elderly populations.

In accordance with the study of McEniery et al., ${ }^{9}$ the present data suggest that aging affects PWV and AI differently. In total, PWV was extremely high (mean PWV $14.4 \mathrm{~m} \mathrm{~s}^{-1}$ ), whereas AI was not (mean value $117 \%$ ). Disassociation between these two hemodynamic factors has been previously described in various populations. ${ }^{10-15}$ Several factors (in separate or in combination) such as: aging process, insulin resistance and metabolic disorders, drugs, as well as associated hemodynamic mechanisms: peripheral vasodilation, attenuation of the reflected wave, shift of the effective reflecting distance, impaired left ventricular function, ${ }^{10}$ might explain this phenomenon. In the present population, the left ventricular function was considerably preserved in both groups (A and B), and cannot be responsible for the outcome. However, it might be explained by peripheral vasodilation, attenuation of the amplitude and later timing of the reflected wave. The proposed mechanisms needs to be further elucidated. Because AI is influenced by both amplitude and timing of the wave reflections, it is difficult to delineate the underlying responsible mechanisms. The application of other types of analysis (for example, wave intensity analysis $)^{16}$ might improve our ability to address this issue in future studies.

Carotid to femoral PWV is considered as a robust marker of CV risk and all-cause mortality ${ }^{17}$ and several studies, as well as a recent metaanalysis, ${ }^{18}$ have proved its ability to predict CV risk in the elderly. ${ }^{3-5}$ Accumulating data, but yet less robust, are available regarding the ability of AI to predict mortality; ${ }^{19-22}$ in the elderly, such data are lacking. In the present study, mortality was independently associated 
Table 5 Models of cox-regression analysis showing the presence of significantly increased mortality (odds ratio) in the presence of both high pulse wave velocity and moderate-to-high augmentation index, after adjustments for potential confounders and risk factors

\begin{tabular}{llc}
\hline & \multicolumn{2}{c}{ Mortality } \\
\cline { 2 - 3 } Adjustment for & Odds ratio $(95 \% \mathrm{Cl})$ & P-value \\
\hline Model 1 (MBP): & $1.81(1.03-3.10)$ & $\mathbf{0 . 0 2 2}$
\end{tabular}

Age, gender, center effect, MBP, HR

Model 2 (mental status):

$1.83(1.08-3.08)$

0.024

Age, gender, center effect, MBP, HR, education level, MMSE

Model 3 (CV disease):

$1.74(1.02-2.95) \quad 0.041$

Age, gender, center effect, MBP, HR,

history of stroke, history of CAD

Model 4 (classical CV risk factors):

$1.84(1.11-3.07)$

0.019

Age, gender, center effect, MBP, HR,

smoking, hypercholesterolemia

Model 5 (cardiac function and structure)

$1.75(1.03-2.98)$

0.038

Age, gender, center effect, MBP, HR,

LV mass, deceleration time, EF

Model 6 (nutrition):

$1.99(1.15-3.44)$

0.014

Age, gender, MBP, HR, center effect,

albumin

Model 7 (renal function):

$1.66(0.97-2.85)$

0.062

Age, gender, MBP, HR, center effect,

creatinine

Abbreviations: $\mathrm{CAD}$, coronary artery disease; $\mathrm{Cl}$, confidence interval; $\mathrm{CV}$, cardiovascular; $\mathrm{EF}$, ejection fraction; HR, heart rate; LV, left ventricle; MBP, mean blood pressure; MMS, mini mental status examination. Center effect: adjustment for possible differences between the participating centers.

Variables in bold letter: significant independent predictors $(P<0.05)$ of mortality.

with age, gender, BP, CV disease and denutrition, but neither with PWV nor with AI, as shown by Kaplan-Meier survival curve analysis. However, those subjects who had combined increased aortic stiffness (third tertile, $\mathrm{PWV}>15.18 \mathrm{~m} \mathrm{sec}^{-1}$ ) and at the same time moderateto-high pressure wave reflections (second and third tertiles, $\mathrm{AI}>112 \%$ ) (group A) had almost double relative risk of mortality, than the rest of the population (group B). Increased mortality in this group was independent from age, gender, BP, history of CVD, denutrition and carotid/cardiac structure-function. In those subjects who presented a 'dissociation' between $\mathrm{PWV}$ and AI, that is, extreme values of aortic stiffness (third tertile, $\mathrm{PWV}>15 \mathrm{~m} \mathrm{~s}^{-1}$ ) with low values of pressure wave reflections (first tertile, $\mathrm{AI}<111 \%$ ), no marked elevation in mortality was observed. Moreover, subjects with high AI (third tertile, AI>127\%) and moderate-to-high PWV (second and third tertiles, PWV $>12.49 \mathrm{~m} \mathrm{sec}^{-1}$ ) had not significantly increased mortality compared with the rest of the population.

The data above imply that there is a synergistic effect on survival when both elevated aortic stiffness and pressure wave reflections are present. The combined effect of PWV and AI on mortality was primarily driven by elevated aortic stiffness, rather than elevated AI. Moreover, this combined effect was not clearly observed when subjects with insulin resistance (consisting almost the $20 \%$ of the population in both group A and group B) or subjects under insulin treatment were excluded. Within the present study, it is not possible to further examine the potential causality of this phenomenon. We have, however, shown previously that in PROTEGER population there is a 'reverse metabolic syndrome, ${ }^{7}$ as low BP, BMI and high insulin sensitivity predict mortality. A similar modulating effect of the presence of metabolic syndrome on the association between CV risk, PWV and AI has been also previously reported. ${ }^{14}$ Moreover, the potential role of renal function has to be elucidated further. It is well described that impaired renal function is associated with arterial stiffening and increase wave reflections, and that both PWV and AI are independent predictors of mortality in end-stage renal disease. In our study, renal function (assessed by plasma creatinine): (1) was not significantly different between group A and group B, (2) was linearly associated with PWV $(r=0.174, P=0.006)$, but not with $\mathrm{AI}$ and (3) was an independent predictor of mortality. Finally, we have to acknowledge the fact that the present sub-analysis of PROTEGER Study may have low statistical power to provide solid results on CV mortality, as only $27 \mathrm{CV}$ deaths were officially established-however, a similar trend was found in the analysis.

In conclusion, the present study provides further insight on the association between large artery stiffness, pressure wave reflections and mortality in the elderly, implying that a synergistic effect of PWV and AI may exist regarding their effect on survival. Future longitudinal studies need to address the following issues: (1) which factors lead to 'disassociation' between aortic stiffness and pressure wave reflections and (2) how this phenomenon might modulate mortality and potentially guide CV risk reduction strategies.

\section{CONFLICT OF INTEREST}

The authors declare no conflict of interest.

\section{ACKNOWLEDGEMENTS}

We are deeply indebted to the PROTEGER patients and their relatives, who made this study possible. Sources of funding: This work was supported by the 'Société Française d'Hypertension Artérielle' and the 'Fondation de France'.

1 Fried LP, Kronmal RA, Newman AB, Bild DE, Mittelmark MB, Polak JF, Robbins JA, Gardin JM. Risk factors for 5 -year mortality in older adults. the cardiovascular health study. JAMA 1998; 279: 585-592.

2 Weverling-Rijnsburger AWE, Blauw GJ, Lagaay M, Knook DL, Meinders E, Westendorp RGJ. Total cholesterol and risk of mortality in the oldest old. Lancet 1997; 350: 1119-1123.

3 Meaume S, Benetos A, Henry OF, Rudnichi A, Safar M. Aortic pulse wave velocity predicts cardiovascular mortality in subjects with $>70$ years of age. Arterioscler Thromb Vasc Biol 2001; 21: 2046-2050.

4 Sutton-Tyrrell K, Najjar SS, Boudreau RM, Venkitachalam L, Kupelian V, Simonsick EM, Havlick R, Lakatta EG, Spurgeon H, Kritchevsky S, Pahor M, Bauer D, Newman A, for the Health $A B C$ Study. Elevated aortic pulse wave velocity, a marker of arterial stiffness, predicts cardiovascular events in well-functioning older adults. Circulation 2005; 111. 3384-3390.

5 Mattace_Raso FUS, van der Cammen TJM, Hofman A, vam Popele NM, Bos ML, Schalekamp ADH, Asmar R, Reneman RS, Hoeks APG, Witteman B, Witteman JCM. Arterial stiffness and risk for coronary heart disease and stroke: The Rotterdam Study. Circulation. 2006; 112: 657-662.

6 Protogerou AD, Safar ME, laria P, Safar H, Le Dudal K, Filipovsky J, Henry O, Dumicetière $\mathrm{P}$, Blacher J. Diastolic blood pressure and mortality in the elderly with cardiovascular disease. Hypertension 2007; 50: 172-180.

7 Vischer UM, Safar ME, Safar H, laria P, Le Dudal K, Henry O, Herrman FR, Ducimtier P, Blacher J. Cardiometabolic determinants of mortality in a geriatric population. Is there a 'reverse metabolic syndrome'? Diabetes Metab 2009; 35: 108-114.

8 Vlachopoulos C, Aznaouridis K, O'Rourke MF, Safar ME, Baou K, Stefanadis C. Prediction of cardiovascular events and all-cause mortality with central haemodynamics: a systematic review and meta-analysis. Eur Heart J 2010; 31: 1865-1871.

9 McEniery CM, Yasmin, Hall IR, Qasem A, Wilkinson IB, Cockcroft JR, ACCT Investigators. Normal vascular aging: differential effects on wave reflection and aortic pulse 
wave velocity: the Anglo-Cardiff Collaborative Trial (ACCT). J Am Coll Cardiol 2005; 46: $1753-1760$.

10 Vyas M, Izzo Jl, Lacourciere Y, Arnold JAO, Dunlap ME, Amato JL, Pfeffer MA, Mitchell GF. Augmentation index and central arterial stiffness in middle-aged to elderly people. Am J Hypertens 2007; 20: 642-647.

11 Vlachopoulos C, Dima I, Aznaouridis K, Vasiliadou C, loakeimidis N, Aggeli C, Toutouza $M$, Stefanadis C. Acute systemic inflammation increases arterial stiffness and decreases wave reflections in healthy individuals. Circulation 2005; 112: 2193-2200.

12 Protogerou AD, Safar ME. Dissociation between central augmentation index and carotid - femoral pulse wave velocity: When and Why? Am J Hypertens 2007; 20: 648-649.

13 Van Bortel LM, Balkestein EJ, van der Heijden-Spek JJ, Vanmolkot FH, Staessen JA, Kragten JA, Vredeveld JW, Safar ME, Struijker Boudier HA, Hoeks AP. Non-invasive assessment of local arterial pulse pressure: comparison of applanation tonometry and echo-tracking. J Hypertens 2001; 19: 1037-1044.

14 Protogerou AD, Blacher J, Aslangul E, Le Jeunne C, Mavrikakis M, Lekakis J, Safar ME. Gender influence on metabolic syndrome's effects on arterial stiffness and pressure wave reflections in treated hypertensive subjects. Atherosclerosis. 2007; 193: $151-158$.

15 Lacy PS, O'Brien DG, Stanley AG, Dewar MS, Swales PPR, Williams B. Increased pulse wave velocity is not associated with elevated augmentation index in patients with diabetes. J Hypertens 2004; 22: 1937-1944.
16 Westerhof BE, Guelen I, Westerhof N, Karemaker JM, Avolio A. Quantification of wave reflection in the human aorta from pressure alone: a proof of principle. Hypertension 2006; 48: 595-601.

17 Laurent S, Cockcroft J, Van Bortel LM, Boutouyrie P, Giannattasio C, Hayoz D, Pannier B, Vlachopoulos Ch, Wilkinson IB, Struijker-Boudier H. Expert consensus document on arterial stiffness: methodological issues and clinical applications. Eur Heart J. 2006; 27: 2588-2605.

18 Vlachopoulos C, Aznaouridis K, Stefanadis C. Prediction of cardiovascular events and all-cause mortality with arterial stiffness: a systematic review and meta-analysis. J Am Coll Cardiol 2010; 55: 1318-1327.

19 London GM, Blacher J, Pannier B, Guerin A, Marchais S, Safar M. Arterial wave reflections and survival in end-stage renal failure. Hypertension 2001; 38: 434-438.

20 Weber T, Auer J, O'Rourke MF, Kvas E, Lassnig E, Lamm G, Stark N, Rammer M, Eber B. Increased arterial wave reflections predict severe cardiovascular events in patients undergoing percutaneous coronary interventions. Eur Heart J 2005; 26: 2657-2663.

21 Chirinos JA, Zambrano JP, Chakko S, Veerani A, Schob A, Willens HJ, Perez G, Mendez AJ. Aortic pressure augmentation predicts adverse cardiovascular events in patients with established coronary artery disease. Hypertension 2005; 45: 980-985.

22 Dart AM, Gatzka CD, Kingwell BA, Willson K, Cameron JD, Liang YL, Berry KL, Wing LM, Reid CM, Ryan P, Beilin LJ, Jennings GL, Johnston $\mathrm{Cl}$, McNeil JJ, Macdonald GJ, Morgan TO, West MJ. Brachial blood pressure but not carotid arterial waveforms predict cardiovascular events in elderly female hypertensives. Hypertension 2006; 47: 785-790. 